\title{
Cardiac effects of acute administration of a protonophore in a rat model
}

\section{AUTHOR(S):}

Minanimo-Muta, Eri; Kato, Takao; Shioi, Tetsuo; Tanada, Yohei; Kimura, Takeshi

\section{CITATION:}

Minanimo-Muta, Eri ... [et al]. Cardiac effects of acute administration of a protonophore in a rat model. Journal of Pharmacy and Pharmacology 2018, 70(8): 1209-1215

\section{ISSUE DATE:}

2018-09

URL:

http://hdl.handle.net/2433/236129

\section{RIGHT:}

This is the peer reviewed version of the following article: [Eri Minanimo - Muta, Takao Kato, Tetsuo Shioi, Yohei Tanada, Takeshi Kimura. Cardiac effects of acute administration of a protonophore in a rat model. Journal of Pharmacy and Pharmacology, 70(9), 1209-1215], which has been published in final form at https://doi.org/10.1111/jphp.12956. This article may be used for non-commercial purposes in accordance with Wiley Terms and Conditions for Use of SelfArchived Versions.; The full-text file will be made open to the public on 06 August 2019 in accordance with publisher's 'Terms and Conditions for Self-Archiving'; This is not the published version. Please cite only the published version.; この 論文は出版社版でありません。引用の際には出版社版をご確認ご利用ください。 
1 Cardiac Effects of Acute Administration of a Protonophore in a Rat Model

3 Eri Minanimo-Muta ${ }^{1}$, Takao Kato ${ }^{1 *}$, Tetsuo Shioi ${ }^{1}$, Yohei Tanada ${ }^{1}$, Takeshi Kimura ${ }^{1}$

$4{ }^{1}$ Department of Cardiovascular Medicine, Graduate School of Medicine, Kyoto University,

554 Kawahara-cho, Sakyou-ku, Kyoto City, Kyoto, Japan

6

7 Corresponding author: Takao Kato

8 Department of Cardiovascular Medicine, Graduate School of Medicine, Kyoto University, 54

9 Shogoin Kawahara-cho, Sakyo-ku, Kyoto, 606-8507 Japan

10 Tel: +81-75-751-3190 FAX: +81-75-751-3203

11 Email: tkato75@kuhp.kyoto-u.ac.jp (TK) 


\section{Abstract}

Introduction: Excessive use of uncoupling agents, previously used as weight-loss agents, has led to the increase of body temperature and death. The aim of the present study was to evaluate the acute cardiac effects of mitochondrial protonophore in a rat model at a high dose, and its specific influence on cardiac substrate uptake.

Methods: Eight-week-old male Sprague-Dawley rats were intraperitoneally injected with the protonophore carbonyl cyanide m-chloro phenyl hydrazone (CCCP; $4 \mathrm{mg} / \mathrm{kg}$ ) or vehicle (dimethyl sulfoxide). Blood pressure, heart rate (HR), and systolic function was recorded. Substrate uptake was monitored by radio-active tracers.

Key findings: Compared to the control group, the respiratory rate and body temperature increased, the left ventricle was dilated, and systolic function transiently deteriorated in the CCCP group. There was no difference in blood pressure and heart rate between the two groups. In cardiac substrate uptake, glucose uptake showed a 95\% increase $(\mathrm{p}<0.05)$, and fatty acid uptake showed a 52\% decrease $(\mathrm{p}<0.05)$ in CCCP-administered group.

Conclusion: The deleterious effects on cardiac function and the changes in substrate uptake were observed when administered with the protonophore at a high dose.

Key words: cardiac function; protonophore; substrate uptake. 


\section{Introduction}

Mitochondria are crucial modulators of viability and death in a variety of cell types, and play important roles in energy production [1-2]. In brown fat cells, mitochondrial respiration is uncoupled from ATP synthesis and heat is produced instead that the energy from betaoxidation is converted into ATP [3]. Uncoupling also occurs to some extent in other cell types. Since the 1940s, several substances including carbonyl cyanide m-chloro phenyl hydrazone (CCCP) and 2,4-dinitrophenol have been known to act as uncoupling agents $[5,6]$. These uncoupling agents have the nature of lipid-soluble acids and provide a bypass pathway of $\mathrm{H}^{+}$across the inner mitochondrial membrane as a protonophore. As a result of this short cut, the proton-motive force is dissipated and ATP cannot be synthesized. Recently, several lines of evidence showed that at low doses, uncouplers reduced reactive oxygen species (ROS) [4,7,8], increased energy expenditure [4,7], and improved longevity [4,8].

Historically, uncoupling agents were used as so-called "weight-loss agents" [9], and their excessive use due to psychological problems has led to the increase of core body temperature and even death [10]. These weight-loss agents are also associated with a danger of overuse due to the image of so-called ideal beauty. The toxic effects of protonophoric mitochondrial uncouplers have been extensively described [10], and the marked toxicity had motivated its withdrawal from the market. However, recent evidences of long administration of chemical 
50

51

uncoupling $[4,7,8]$ make the agent began to show sings coming life again, for example, as a patent of the new derivatives for the use of non-alcoholic fatty liver disease. However, detailed knowledge of the effects of their acute administration at a high dose to the heart, which is one of most energy-consuming organs, have not been examined.

We previously reported the uptake of the radioisotope-labeled tracer technetium $99 \mathrm{~m}$ Technetium $\left({ }^{99 \mathrm{~m}} \mathrm{Tc}\right)$-sestamibi (MIBI) signals in the perfused and excised heart in a rat model administered a protonophore, CCCP [11]. In the excised hearts of Sprague-Dawley (SD) rats administered ${ }^{99} \mathrm{~m}$ Tc-MIBI which is positively charged and distributed into mitochondria according to the mitochondrial membrane potentials, CCCP decreased the ${ }^{99 \mathrm{~m}} \mathrm{Tc}-\mathrm{MIBI}$ signals along with a decrease of in situ ATP and phosphocreatine contents of the heart. In the present study, we aimed to clarify the acute effects of the protonophore on cardiac function and cardiac substrate uptake in a rat model, which are the novel points of the present study. 


\section{Methods}

\section{Animals and materials}

Eight-week-old male SD rats (body weight 280-290 g) were administered CCCP (Wako Pure

Chemical Industries; Osaka, Japan). Animal care and experimental procedures were approved by the Institutional Animal Care and Use Committee of Kyoto University (permission no.

MedKyo14184) and conducted following the Guide for Care and Use of Laboratory Animals published by the United States National Institutes of Health. ${ }^{99 m}$ Tc-MIBI and ${ }^{125} \mathrm{I}-(\mathrm{p}-$ iodophenyl)-9-R,S-methylpentadecanoic acid (9MPA) were purchased from FUJIFILM RI Pharma Co. Ltd. (Tokyo, Japan). ${ }^{18}$ F-deoxyglucose (FDG) was synthesized by Kyoto University Hospital. CCCP (Wako Pure Chemical Industries, Osaka, Japan) was diluted in 100\% dimethyl sulfoxide (DMSO, Wako Pure Chemical Industry, Osaka, Japan) to prepare a $10 \mathrm{mM}$ stock solution.

\section{Physiological and hemodynamic analysis}

Protocol 1: To investigate the physiological and hemodynamic changes in CCCPadministered rats, 8 -week-old male SD rats $(n=6)$ were intraperitoneally injected with $\mathrm{CCCP}$ $(4 \mathrm{mg} / \mathrm{kg})$ or vehicle (DMSO; $\mathrm{n}=6)$. Body temperature and blood pressure was recorded at the rectum at 30 min after the CCCP injection (AD-1687, A\&D Company Ltd., Tokyo, Japan). Blood pressure is determined by the tail-cuff method using a noninvasive automated blood 
83

pressure apparatus (Softron SBP-200, Softron Co. Ltd., Tokyo, Japan) without anesthesia.

Transthoracic echocardiographic analysis was performed as previously reported [12] using a Sonos-5500 echocardiograph (Agilent Technologies, Santa Clara, CA, USA) with a 15-MHz linear transducer. Heart rate (HR), intraventricular septal thickness (IVSd), left ventricular dimension in the diastolic phase (LVDd), and left ventricular dimension in the systolic phase (LVDs) were measured with M-mode echocardiography $30 \mathrm{~min}, 90 \mathrm{~min}$, and $180 \mathrm{~min}$ after CCCP injection, and fractional shortening (FS) was calculated using the following formula: $\% \mathrm{FS}=[(\mathrm{LVDd}-\mathrm{LVDs}) / \mathrm{LVDd}] \times 100$.

\section{Effect of CCCP on the uptake of a glucose and fatty acid radiotracer}

Protocol 2: To analyze the effect CCCP on glucose and fatty acid uptake, ${ }^{18} \mathrm{~F}$-deoxyglucose

(FDG) and ${ }^{125}$ I-9MPA was used, respectively. The rats ( $n=6$ per group) were fasted overnight, administered CCCP, and injected with $1 \mathrm{mCi}$ of ${ }^{18} \mathrm{FDG}$ and $20 \mu \mathrm{Ci}$ of ${ }^{125} \mathrm{I}-9 \mathrm{MPA}$ simultaneously 45 min later. They were euthanized by decapitation 45 min after the injection, and the hearts were removed and washed in cold saline. The 1/3 portion of the apical side was frozen in liquid nitrogen and the radioisotopic activity was measured using a scintillation counter (Cobra2 ${ }^{\mathrm{TM}}$ Auto-gamma, Packard) $[13,14]$. To measure ${ }^{18}$ FDG uptake, radioisotopic activity was measured just after euthanization because the half-decay time of ${ }^{18} \mathrm{FDG}$ is 110 min. To measure ${ }^{125} \mathrm{I}-9 \mathrm{MPA}$ uptake, radioisotopic activity was measured $48 \mathrm{~h}$ after the 
102 euthanization. The myocardial uptake levels of ${ }^{18} \mathrm{FDG}$ or ${ }^{125} \mathrm{I}-9 \mathrm{MPA}$ were assessed by direct

103

104

105

106

107

108

109

110

111

112

113

114

115

116

measurement using the scintillation counter. The amount of radioisotope incorporated is

expressed as a percentage of the administered radioisotope activity corrected by heart weight

(g). Cross-talk between the two tracers was negligible [13, 14].

\section{Washout of ${ }^{99 m}$ Tc-MIBI in vivo}

Protocol 3: In order to investigate the mechanism of substrate change, we calculated ${ }^{99 \mathrm{~m}} \mathrm{Tc}-$

MIBI in vivo to show the changes in mitochondrial function $[15,16,17]$. A dose of $15 \mathrm{MBq}$

$(405.4 \mu \mathrm{Ci})$ of ${ }^{99 \mathrm{~m}} \mathrm{Tc}-\mathrm{MIBI}$ was injected into the tail vein under anesthesia with pentobarbital

sodium (10 mg/kg IP). Rats were placed exactly $10 \mathrm{~cm}$ from the collimator. Pre-CCCP or

vehicle-administered images $(64 \times 64$ matrix size $)$ were obtained 15 min after the ${ }^{99 m}$ Tc-MIBI

injection. Then, CCCP or vehicle was administered intraperitoneally to rats $90 \mathrm{~min}$ after the

${ }^{99 m}$ Tc-MIBI injection (CCCP: $\mathrm{n}=8$, vehicle: $\mathrm{n}=7$ ). Thereafter, post-CCCP or vehicle-

administered images were obtained $180 \mathrm{~min}$ after the ${ }^{99 \mathrm{~m}} \mathrm{Tc}-\mathrm{MIBI}$ injection. To calculate the

rate of myocardial ${ }^{99 \mathrm{~m}} \mathrm{Tc}-\mathrm{MIBI}$ washout following injection, a region of interest was manually

drawn around the heart and in the mediastinum area between the upper limbs. The myocardial

${ }^{99 \mathrm{~m}} \mathrm{Tc}-\mathrm{MIBI}$ washout rate (percentage) was calculated using the following equation: (A -

$\mathrm{B} * \mathrm{DC}) / \mathrm{A} \times 100(\%)$, in which A was defined as (pre-CCCP or vehicle-administered heart

count - pre-CCCP or vehicle-administered mediastinum count), B was defined as (post-CCCP 
121 or vehicle-administered heart count - post-CCCP or vehicle-administered mediastinum

122 count), and DC is the decay coefficient. [15, 16, 17]

123

124

125 Statistical analysis

126 All data are expressed as the mean \pm standard error of the mean (SEM). Differences between

127 the groups were compared using the Kruskal-Wallis post-hoc using Dunn's test. In all tests, a

128 value of $\mathrm{p}<0.05$ was considered statistically significant.

129 


\section{Results}

Effects of CCCP on body temperature, hemodynamics, and cardiac function

Body temperature analyzed at 30 min after the CCCP injection was higher than that after

vehicle injection $(\mathrm{p}=0.024$, Figure $1 \mathrm{~A})$, indicating that electron transport was uncoupled by

CCCP. There was no difference in heart rate between CCCP-administered and vehicle-

administered rats (Figure 1B). Blood pressure tended to decrease in CCCP-administered rats

examination showed that both LVDd and LVDs increased $(\mathrm{p}=0.0048$ and 0.0047 ,

injection (Figures 1D, 1E, and 1F, respectively), indicating that CCCP caused transient left LV administered rats was diminished at 90-180 min after the CCCP injection.

\section{CCCP changed substrate uptake in the cardiac tissue}

144 Next, we examined whether CCCP caused a change in the myocardial uptake of glucose and

145 fatty acids using ${ }^{18}$ FDG and ${ }^{125}$ I-9MPA, respectively (Figure 2A). Compared to the vehicle

146 group, glucose uptake showed a 95\% increase $(p=0.033)$ and the fatty acid uptake showed a

$14752 \%$ decrease $(\mathrm{p}=0.033) 90$ min after CCCP administration (Figures $2 \mathrm{~B}$ and $2 \mathrm{C}$, respectively), indicating that the protonphore caused changes in substrate uptake. 
151 To investigate the effect of CCCP on membrane potentials in vivo, we obtained pre-CCCP or

152 vehicle-administered images 15 min after the ${ }^{99 m}$ Tc-MIBI injection, then CCCP or vehicle

153 was injected, and post-CCCP or vehicle-administered images were obtained 180 min after the

155 decreased after the CCCP injection (Figure 3B, lower panels) compared to that in vehicle-

156 administered rats (Figure 3B, upper panels). The analysis of in vivo images showed that the

157 washout rate of ${ }^{99 m}$ Tc-MIBI was significantly increased in CCCP rats $(p=0.015$; Figure 3C). 


\section{Discussion}

In summary, CCCP caused transient LV dilatation and systolic dysfunction. CCCP increased glucose uptake, and decreased fatty acid uptake in the rat heart tissue and ${ }^{99 \mathrm{~m}} \mathrm{Tc}-\mathrm{MIBI}$ washout rate in vivo.

We recently reported that the accumulation of ${ }^{99 \mathrm{~m}} \mathrm{Tc}-\mathrm{MIBI}$ signals was correlated to the tetramethylrhodamine ethyl ester assay in ex vivo perfused rat hearts [11]. We found that CCCP decreased the in situ ATP levels at 30 min after the injection [11], suggesting that energy deficiency might cause the LV dilatation and systolic dysfunction observed in the present study. This mechanism of the cardiac dysfunction is currently only speculative and was not directly elucidated in the present study; however, the effects of CCCP were transient according to the metabolic rate of CCCP. Dillis et al. [18] reported that hepatic ATP and cosubstrate levels decreased $30 \mathrm{~min}$ after CCCP injection and returned to normal at $60 \mathrm{~min}$ after the injection, which is consistent with the results of the present study. ${ }^{9 \mathrm{~m}} \mathrm{Tc}-\mathrm{MIBI}$ has a high affinity for the negative charges associated with membrane potentials across the mitochondrial membrane, according to the Nernstian equation [19,20]. A blood clearance study showed that myocellular equilibrium was reached at a $\mathrm{t}_{1 / 2}$ of $2-5 \mathrm{~min}$ in clinical use [21]. Therefore, the washout rate was increased according to the decreased membrane potentials. The observed increase in ${ }^{99 \mathrm{~m}} \mathrm{Tc}-\mathrm{MIBI}$ washout rate in the present study has the 
178

possibility to represent, at least partly, a decrease in mitochondrial membrane potentials and dysfunction of mitochondria which support the CCCP-induced changes of the substrate uptake (Figure 2) and energy deficiency [11] in the heart.

${ }^{18}$ FDG uptake increased in the present study. Although neither the metabolic rate of glycolysis nor the molecular mechanism for directly increasing ${ }^{123} \mathrm{FDG}$ uptake was examined in the present study, a possible mechanism of this rapid regulation is adenosine monophosphate (AMP)-activated protein kinase. An increase of the AMP to ATP ratio, i.e. energy deficiency, activated AMP-activated protein kinase and enhanced glucose uptake and glycolysis [22]. By contrast, the uptake of ${ }^{123}$ I-9MPA decreased. ${ }^{123}$ I-9MPA was rapidly metabolized to iodophenyl-3-methylnonanoic acid (3MNA) by beta-oxidation, and was not further metabolized [23,24]; therefore, it is generally considered to reflect fatty acid oxidation in mitochondria [23,24]. Ikawa et al. reported the increased iodine-123-labelled 15-(piodophenyl)-3-(R,S)-methylpentadecanoic acid ( $\left.{ }^{123} \mathrm{I}-\mathrm{BMIPP}\right)$, another tracer of fatty acids, in patients with mitochondrial cardiomyopathy with the increase in ${ }^{99} \mathrm{~m}$ Tc-MIBI washout ratio [25]. Most of the ${ }^{123}$ I-BMIPP was incorporated into the triglyceride pool, and reflects the turnover of the triglyceride pool in the cytosol [26]. In patients with mitochondrial cardiomyopathy, the energy production shifts from the metabolism of fatty acids to the glycolytic pathway with the excess of glycerol-3-phosphate, leading to the enhanced synthesis 
197 of triglycerides. Thus, in chronic mitochondrial failure, ${ }^{123}$ I-BMIPP is incorporated more into

triglyceride-pool and remains in triglyceride pool in the cytosol [26]. Thus, decreased uptake of ${ }^{123}$ I-9MPA reflects the acute mitochondrial dysfunction, and increased uptake (and decreased washout) of ${ }^{123}$ I-BMIPP reflects the chronic mitochondrial dysfunction.

Life-long administration of low-dose chemical uncoupling 2,3-dinitrophenol to mice caused no adverse effects, decreased body weight, and prolonged survival [4][ 27]. However, the dose used in the present study caused LV dysfunction. One report showed that CCCP decreased hepatic ATP production when administered to rats at a dose of $4 \mathrm{mg} / \mathrm{kg}$ with no mortality, whereas a dose of $5 \mathrm{mg} / \mathrm{kg}$ resulted in $11 \%$ mortality [18]. The LD50 was found to be approximately $8 \mathrm{mg} / \mathrm{kg}$. Hence, the dose of CCCP used in this short-term experiment is thought to be relatively high, indicating that high-dose CCCP is detrimental for cardiac function. The next key question is to determine to what dosage and for how long uncoupling would have to be increased to achieve beneficial effects due to decreased ROS production and to avoid detrimental effects due to decreased ATP production and heart failure.

\section{Limitations}

The limitations of the present study include the lack of an observed dose-response and the lack of a clear mechanism to explain the observed effects. Although the relationship between 
${ }^{99} \mathrm{~m}$ Tc-MIBI accumulation and mitochondrial potential was assessed in cultured myocytes

217 [28], direct monitoring of the mitochondrial potential in vivo was also difficult to achieve;

218 however, further studies about serial measurements of the phosphocreatine and $\beta$ ATP levels in

219 vivo would provide useful information on the energy deficiency and its recovery in this

220 model. Lack of measuring oxygen consumption rate in vivo is another limitation. Finally, we

221 acknowledged that this work was a subsequent series of studies using CCCP [11], although

222 the data in this work provided the insights on the changes in substrate uptake and function

223 when we used CCCP.

224

225 Conclusions

226 The deleterious effects on cardiac function and the changes in substrate uptake were observed

227 when administered with the protonophore at a high dose.

\section{Competing interest}

229 None declared.

\section{$230 \quad$ Founding}

231 This work was supported by grants from the Japan Society for the Promotion of Science

232 (15K19400).

\section{Acknowledgments}

234 The founders had no the role in design, in the collection, analysis, and interpretation of data; 
235 in the writing of the manuscript; and in the decision to submit the manuscript for publication. 


\section{References}

1. Green DR, Kroemer G. The pathophysiology of mitochondrial cell death. Science 2004; 305: 626-629.

2. Neubauer S. The failing heart--an engine out of fuel. $N$ Engl J Med. 2007; 356: 1140-1151.

3. Cannon B, Nedergaard J. Neither brown nor white. Nature. 2012; 488: 286-287.

4. Caldeira da Silva CC et al. Mild mitochondrial uncoupling in mice affects energy metabolism, redox balance and longevity. Aging Cell. 2008; 7: 552-60.

5. Lipschitz WL, Bueding E. Mechanism of the biological formation of conjugated glucuronic acids. J Biol Chem. 1948; 129: 333-358.

6. Loomis WF, Lipmann F. Reversible inhibition of the coupling between phosphorylation and oxidation. J Biol Chem 1948; 174 : 807.

7. Goldgof $\mathrm{M}$ et al. The chemical uncoupler 2,4-dinitrophenol (DNP) protects against dietinduced obesity and improves energy homeostasis in mice at thermoneutrality. J Biol Chem. 2014; 289: 19341-19350.

8. Perrino $\mathrm{C}$ et al. Genetic deletion of uncoupling protein 3 exaggerates apoptotic cell death in

252 the ischemic heart leading to heart failure. J Am Heart Assoc. 2013; 2: e000086. 
256

257

258

259

260

261

262

263

264

265

266

267

268

269

270

271

272

273

274

11. Kawamoto A et al. Measurement of technetium-99m sestamibi signals in rats administered a mitochondrial uncoupler and in a rat model of heart failure. PLoS One. 2015; 10: e0117091.

12. Tanada Y et al. Branched-chain amino acids ameliorate heart failure with cardiac cachexia in rats. Life Sci. 2015; 137: 20-27.

13. Unno K et al. Relation of functional and morphological changes in mitochondria to myocardial contractile and relaxation reserves in asymptomatic to mildly symptomatic patients with hypertrophic cardiomyopathy. Eur Heart J. 2009; 30:1853-1862.

14. Kato T et al. Analysis of metabolic remodeling in compensated left ventricular hypertrophy and heart failure. Circ Heart Fail 2010; 3: 420-430.

15. Kato T. A data sheet for the simultaneous assessment of dual radiotracer uptake in the heart. MethodsX. 2016; 3: 289-296.

16. Matsuo $\mathrm{S}$ et al. A novel clinical indicator using tc-99m sestamibi for evaluating cardiac mitochondrial function in patients with cardiomyopathies. J Nucl Cardiol. 2007; 14: 215-220.

17. Kato $\mathrm{T}$ et al. A potential linkage between mitochondrial function of the heart and leg muscles in patients with heart failure. Int J Cardiol. 2015; 188:67-69.

18. Dills RL, Klaassen CD. The effect of inhibitors of mitochondrial energy production on hepatic glutathione, UDP-glucuronic acid, and adenosine 3'-phosphate-5'-phosphosulfate concentrations. Drug Metab Dispos. 1986; 14: 190-196.

19. Piwnica-Worms D et al. Uptake and retention of hexakis (2-methoxyisobutyl isonitrile) 
275 technetium(I) in cultured chick myocardial cells. Mitochondrial and plasma membrane

276 potential dependence. Circulation 1990; 82: 1826-1838.

277

20. Backus M et al. Microprobe analysis of Tc-MIBI in heart cells: calculation of mitochondrial membrane potential. Am J Physiol 1993; 265: C178-187.

21. Wackers FJ et al. Technetium-99m hexakis 2-methoxyisobutyl isonitrile: human biodistribution, dosimetry, safety, and preliminary comparison to thallium-201 for myocardial perfusion imaging. J Nucl Med 1989; 30: 301-311.

22. Long YC, Zierath JR. AMP-activated protein kinase signaling in metabolic regulation. $J$ Clin Invest. 2006; 116: 1776-1783.

23. Knapp FF, Kropp JJ. Iodine-123-labelled fatty acids for myocardial single-photon emission tomography: current status and future perspectives. Eur J Nucl Med 1995; 22: 361381.

24. Watanabe $\mathrm{K}$ et al. Constitutive regulation of cardiac fatty acid metabolism through peroxisome proliferator-activated receptor alpha associated with age-dependent cardiac toxicity. J Biol Chem 2000; 275: 22293-22299.

25. Ikawa $\mathrm{M}$ et al. Evaluation of respiratory chain failure in mitochondrial cardiomyopathy by assessments of 99mTc-MIBI washout and 123I-BMIPP/99mTc-MIBI mismatch. Mitochondrion. 2007;7:164-170.

26. Morishita $\mathrm{S}$ et al. Kinetics of radioiodinated species in subcellular fractions from rat hearts 
294 following administration of iodine-123-labelled 15-(p-iodophenyl)-3-(R,S)-

295 methylpentadecanoic acid (123I-BMIPP). Eur J Nucl Med. 1996;23:383-9

296 27. Geisler JG. Targeting energy expenditure via fuel switching and beyond. Diabetologia

$297 \quad 2011 ; 54: 237-244$.

298 28. Chiu ML et al. Effect of mitochondrial and plasma membrane potentials on accumulation

299 of hexakis (2-methoxyisobutylisonitrile) technetium(I) in cultured mouse fibroblasts. $J$ Nucl

$300 \quad$ Med. 1990; 31: 1646-1653.

301 
302

303

304

305

306

307

308

309

310

Figure legends

Figure 1: Physiological and echocardiographic examination of CCCP-administered rats

(A) Body temperature analyzed at 30 min after the CCCP injection was higher than that after vehicle injection. (B) Heart rate did not differ between CCCP-administered and vehicleadministered rats (Vehicle: $n=6$, CCCP: $n=6$ ). (C) Blood pressure tended to decrease in CCCP-administered rats $(116 \pm 4 \mathrm{mmHg})$ compared to vehicle-administered rats $(131 \pm 3$ mmHg). Vehicle: $n=6$, CCCP: $n=6$. (D) Left ventricular diastolic dimension (LVDd).

Vehicle: $n=6$, CCCP: $n=6$. (E) Left ventricular systolic dimension (LVDs). (F) Fractional shortening (FS). Serial echocardiographic examination showed that both LVDs and LVDd increased and FS decreased up to 60 minutes after the CCCP injection. All circles and bars indicate means and SEMs respectively. ${ }^{*} \mathrm{p}<0.05$ versus vehicle-administered rats.

\section{Figure 2: The uptake of ${ }^{18}$ FDG was increased and the uptake of ${ }^{125}$ I-9MPA was}

\section{decreased by CCCP.}

(A) A schema of the study for analyzing the extracted hearts. (B) and (C) The uptake of

${ }^{18}$ FDG and ${ }^{125}$ I-9MPA, respectively. All bars indicate means and SEMs. ${ }^{*} \mathrm{p}<0.05$ versus vehicle-administered rats. $\mathrm{n}=6$ in each group.

Figure 3: ${ }^{99 m}$ Tc-MIBI washout was increased in rats administered CCCP 
321 (A) A schema of the study for analyzing the images and extracted hearts. (B) Representative

322 in vivo images of ${ }^{99 \mathrm{~m}} \mathrm{Tc}-\mathrm{MIBI}$ distribution. Myocardial retention of ${ }^{99 \mathrm{~m}} \mathrm{Tc}-\mathrm{MIBI}$ was markedly

323 decreased after the CCCP injection (lower panels) compared to vehicle-administered rats

324 (upper panels). White arrowheads indicate hearts. (C) Analysis of in vivo images showed that

325 the ${ }^{99 \mathrm{~m}} \mathrm{Tc}-\mathrm{MIBI}$ washout rate was significantly increased in CCCP rats (Vehicle: $\mathrm{n}=7$, CCCP:

$326 \mathrm{n}=8)$. WR, washout rate. All bars indicate means and SEMs. * $\mathrm{p}<0.05$ versus vehicle-

327 administered rats.

328 
B

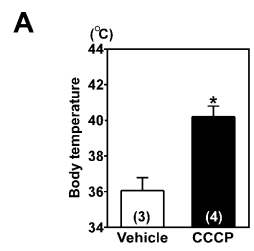

C
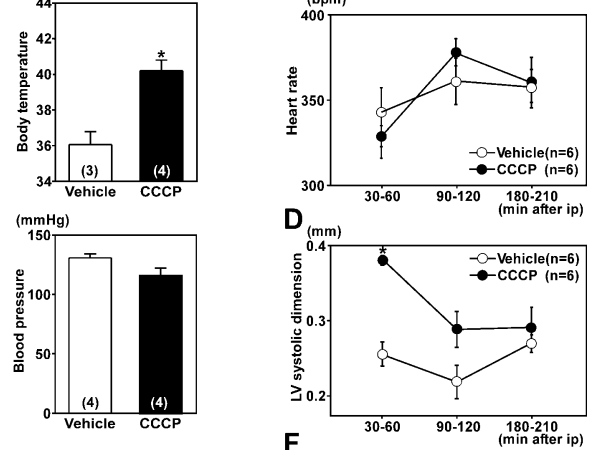

E
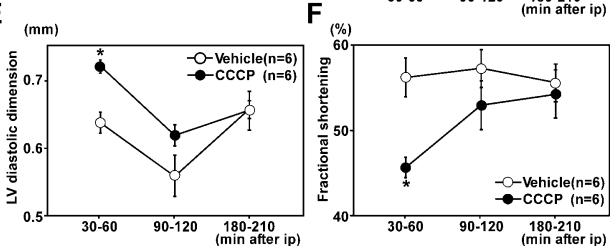

329

Figure 1

331 
A

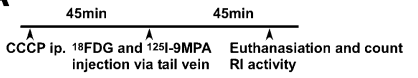

CCCP ip. 18FDG and 1251-9MPA Euthanasi

B (\%administered $\quad$ C (\%administered

332

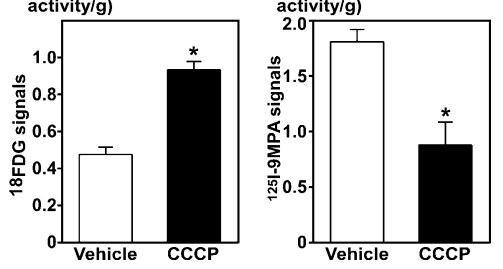

333 Figure 2

334 
A

${ }^{99 m} \mathrm{TC}-\mathrm{MIBI}$ injection

CCCP

\begin{tabular}{cccc}
$\Downarrow 15 \min$ & $\Downarrow$ & 3 hour \\
\hline$\uparrow$ & $90 \min$ & $\uparrow$
\end{tabular}

\begin{tabular}{|c|}
\hline Before vehicle or CCCP IP \\
$99 \mathrm{~m}$ Tc-MIBI image acqusition \\
$99 \mathrm{~m}$ Tc-MIBI image acqusition \\
\hline
\end{tabular}
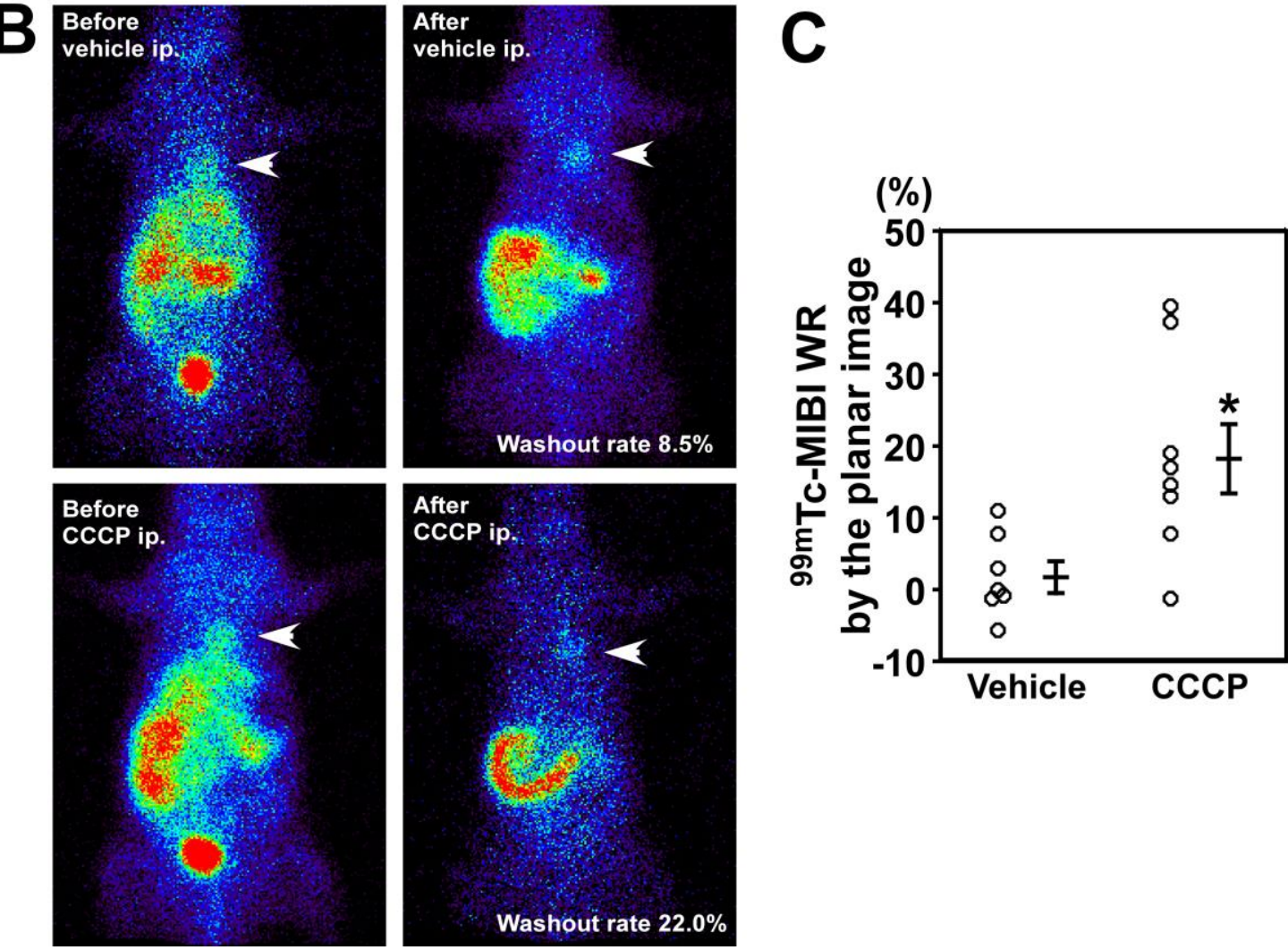

$336 \quad$ Figure 3 